\title{
Molecular Tailoring of Surfaces via Pulsed Plasma Polymerization
}

\author{
Haibo Qiu ${ }^{1}$, F. Samuel Sanchez-Estrada ${ }^{2}$ and Richard B. Timmons ${ }^{3}$ \\ ${ }^{1}$ Materials Science and Engineering Program; ${ }^{2}$ Environmental Science and Engineering Program; \\ ${ }^{3}$ Department of Chemistry and Biochemistry, University of Texas at Arlington, Arlington, TX 76019, USA
}

\begin{abstract}
The use of a variable duty cycle pulsed plasma, in lieu of the conventional continuous-wave operational mode, is employed to provide enhanced film chemistry control during polymer film formation. This film chemistry control is illustrated with studies of perfluorocarbon polymers and by synthesis of electrically conductive films from pyrrole. The ability to synthesize polymer films under very low power inputs, using the pulsed technique, permits generation of relatively linear polymeric structures. Contrasts in several important plasma variables under continuous and pulsed modes are identified and discussed in terms of their influence in providing added film chemistry control under pulsed conditions
\end{abstract}

Keywords: plasma polymerization, pulsed plasma, linear polymers, film chemistry control

\section{Introduction}

Plasma polymerizations represent an increasingly popular and relatively facile route to surface modification [1]. Early work in this field emphasized the unique film compositions attainable under continuous-wave $(\mathrm{CW})$ plasma operational conditions [2]. During typical high energy input $\mathrm{CW}$ plasma polymerizations, relatively highly cross-linked structures are obtained, in many cases these film compositions having little resemblance to that of the monomers from which they are formed.

In contrast with these earlier studies, a major portion of the more recent plasma polymerization literature has focused on acquiring improved film chemistry control during polymer formation. Such film chemistry controllability is highly desired for applications in which surface chemical compositions play the pivotal role in dictating device performance (e.g. sensors, catalysts, biomaterials, etc.). In terms of plasma polymerization derived surface modifications, one would like to achieve this molecular tailoring capability while retaining the numerous advantages inherently available with this coating technology. These include an all-dry, one-step, relatively rapid process that provides adhesive, pin-hole free, conformal films of uniform thickness [3].

Film chemistry controllability under $\mathrm{CW}$ plasma condition has been examined with systematic changes in various operational variables. These variables include monomer flow rate and pressure; power input; substrate location and temperature. In particular, many studies have emphasized variations in film compositions with changes in the composite variable W/FM, where W is the power input, $F$ is the flow rate and $M$ the molecular mass of the monomer [2]. Although variations in film compositions are observed with changes in the W/FM variable, the ranges of compositions are frequently restricted by formation of oily or powdery deposits at low power inputs and by lack of film formation (i.e. dominance of ablation reactions) at high power inputs.

Recently, we have examined the utility of a pulsed plasma, in lieu of the traditional CW operational mode, as a possible alternate approach to achieve the much desired film chemistry controllability. In fact, large-scale and progressive changes in film compositions, obtained from a given monomer, have been observed with sequential variations in the pulsed plasma duty cycle employed, all other plasma variables being held constant [3-5]. Subsequently this film chemistry controllability has been demonstrated with a range of monomers, including highly reactive compounds [6,7], organometallics $[8,9]$ and mixtures [10].

The present paper involves an extension of these studies, with particular emphasis on 
contrasting differences in dynamic variables under $\mathrm{CW}$ and pulsed plasma conditions and the influence of these variables on film chemistry compositional control issues.

\section{Experimental}

The apparatus and general procedures employed in operation of the $13.56 \mathrm{MHz}$ RF pulsed-plasma reactor have been described previously [11]. The plasma coated substrates were characterized using XPS and FT-IR analysis. Film thicknesses employed $(>100 \mathrm{~nm})$ were sufficiently thick to prevent substrate contributions to the XPS spectra. Film thicknesses were determined by surface profilometry.

Plasma polymerizations were carried out using monomer pressures in the range of 30 to 150 millitorr, and typical flow rates of 0.2 to $5.0 \mathrm{~cm}^{3}$ (STP/min). For each monomer, comparative analysis of film compositions, as a function of plasma duty cycles were carried at a fixed pressure and fixed flow rate. Typical peak power inputs of 50 to 300 watts were employed as noted in the next section.

An important feature of the pulsed-plasma approach is that it affords an opportunity to generate plasma film under unusually low total power inputs. The equivalent power $\left(\mathrm{W}_{\mathrm{eq}}\right)$ under pulsed conditions is calculated from the equation:

$$
\mathrm{W}_{\mathrm{eq}}=\tau_{\mathrm{on}} /\left(\tau_{\mathrm{on}}+\tau_{\text {off }}\right) \cdot(\text { Peak Power })
$$

where $\tau_{\text {on }}$ and $\tau_{\text {off }}$ are the plasma on and off times, and peak power is the power input during the plasma on periods of the pulsed cycle. In effect, the pulsed approach permits film formation under average power inputs which are significantly lower than those which are employable under $\mathrm{CW}$ conditions [7]. For example, we can generally obtain high quality film formation under pulsed conditions at average power inputs which are an order of magnitude lower than those available under $\mathrm{CW}$ conditions. In effect, this permits an extension of the W/FM studies to a much wider range than available under $\mathrm{CW}$ conditions. Interestingly, this extension of plasma polymer formation to very low power inputs under pulsed conditions is achieved without undo compromises of film deposition rates, as documented below.

\section{Results}

The effects of variations in pulsed plasma duty cycles on polymer film compositions are shown in Figure 1 and 2 for the monomers perfluorohexane $\left(\mathrm{C}_{6} \mathrm{~F}_{14}\right)$ and pyrrole $\left(\mathrm{C}_{4} \mathrm{H}_{5} \mathrm{~N}\right)$. In the case of $\mathrm{C}_{6} \mathrm{~F}_{14}$, a variation from a highly-cross linked film to a relatively linear fluorocarbon polymer was obtained as the plasma duty cycle employed during film formation was decreased. The sequential changes in film compositions are clearly evidenced in the C (1s) high resolution XPS spectra shown in Figure 1 . Here the plasma duty cycles employed during film formation are indicated by a simple ratio of plasma on to plasma off times (in $\mathrm{ms}$ ).

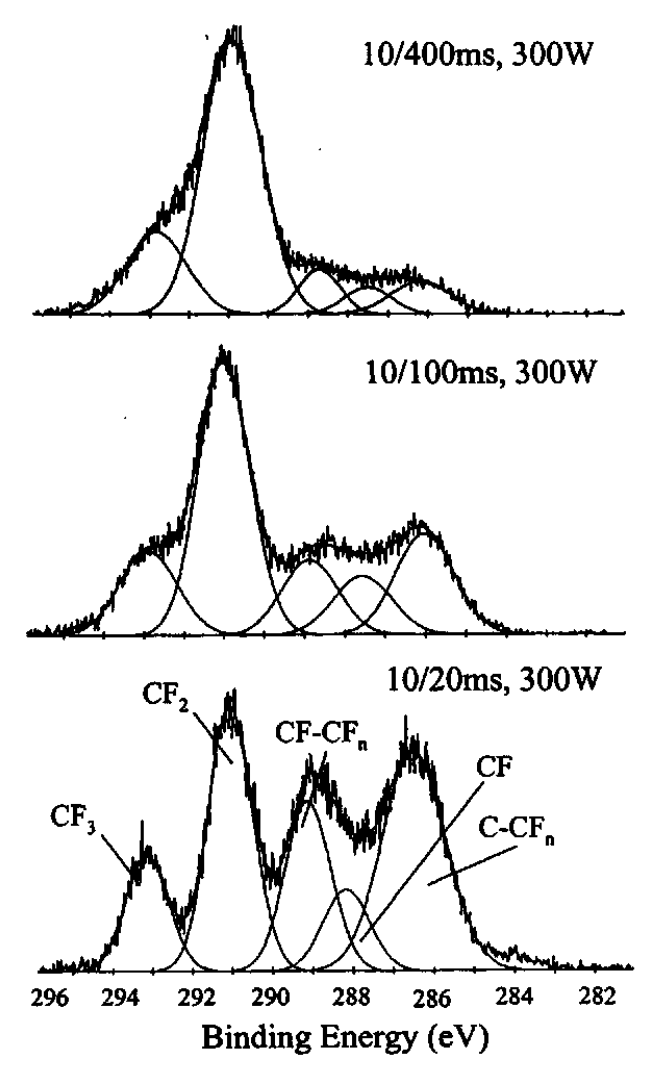

Figure 1. Variation in the $\mathrm{C}(1 \mathrm{~s})$ high resolution XPS spectra of films obtained from $\mathrm{C}_{6} \mathrm{~F}_{14}$ monomer as a function of the pulsed plasma duty cycle employed during polymerization. All pulsed runs were carried out at a peak power input of 300 $\mathrm{W}$ and on/off time (in ms) as shown.

FT-IR absorbance spectra of films obtained in the plasma polymerization of pyrrole are shown in Figure 2. Spectra of films obtained under pulsed plasma conditions of $10 / 100 \mathrm{~ms}, 25 \mathrm{~W}$ and $\mathrm{CW}$ $25 \mathrm{~W}$ are shown. Of particular significance is the increased prominence of the $\mathrm{N}-\mathrm{H}$ stretching ( $\left.3400 \mathrm{~cm}^{-1}\right)$; and $\mathrm{C}-\mathrm{H}$ aromatic ring bending $(\sim 750$ $\mathrm{cm}^{-1}$ ) vibrations in the pulsed plasma generated films relative to those obtained in the $\mathrm{CW}$ deposited film. Clearly, the pulsed plasma generated film exhibits a much higher level of ring 


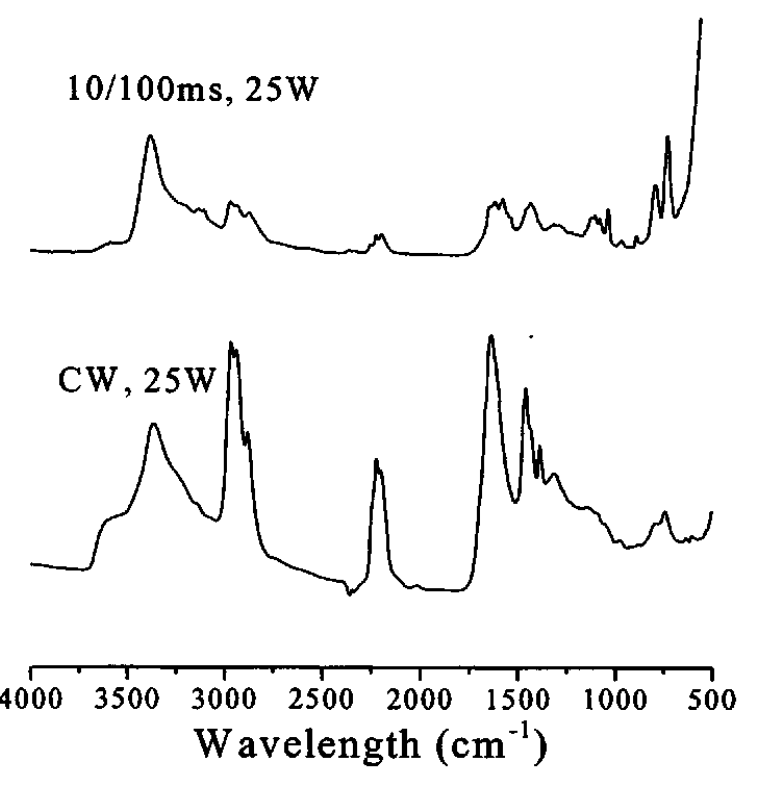

Figure 2. Comparison of FT-IR absorbance spectra of polymer films obtained from pyrrole under pulsed (top) and CW (bottom) conditions.

retention than that obtained under $\mathrm{CW}$ deposition conditions. A higher level of ring retention results in formation of a more linear polymeric structure. This more highly linear structure is confirmed by sharp increase in the electrical conductivity of these films as the plasma duty cycle employed during their synthesis is reduced. The conductivity of these films, after appropriate doping with iodine, are shown in Figure 3 as a function of the equivalent power employed during film deposition. The sharp increases in film conductivities deposited at the lowest pulsed plasma power input confirms the remarkably high retention of monomer identity in these polymers. Overall, both the extent of retention of ring structure and film conductivities are significantly higher than earlier studies of pyrrole polymerization under $\mathrm{CW}$ conditions $[12,13]$.

Finally, an interesting feature of pulsed plasma polymerizations is the sharply increased film formation energy efficiency observed with various monomers [14]. A particularly dramatic example of this increased energy efficiency under progressively lower duty cycles is shown in Figure 4. In this study, the plasma polymerization of tetramethlysilane (TMS) was examined under CW and various pulsed conditions. The average power input was maintained constant in each run, as can be computed from the data shown in Figure 4. Clearly, a dramatically increased energy efficiency is observed in film formation rates as the

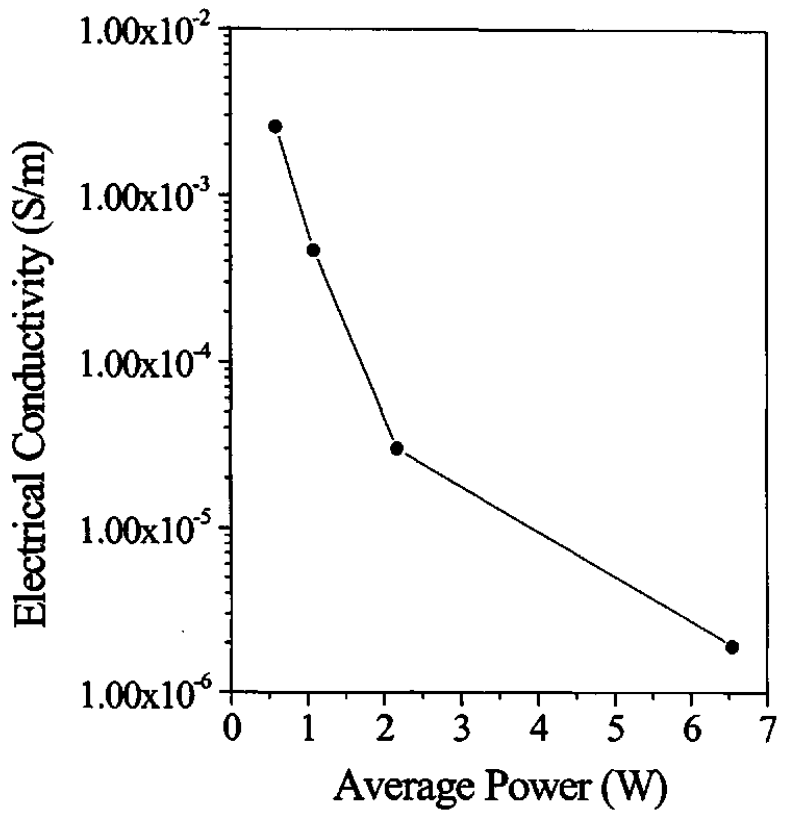

Figure 3. Variation in the electrical conductivity of $\mathbf{I}_{2}$ doped pulsed plasma polymerized pyrrole films as a function of average power employed during film formation.

equivalent power (i.e. duty cycle) is reduced, as expressed in terms of film thickness per Joule of energy input. We have interpreted this increased energy efficiency in film formation with decreasing plasma duty cycles as clear evidence for significant polymer formation during plasmaoff times under pulse deposition conditions.

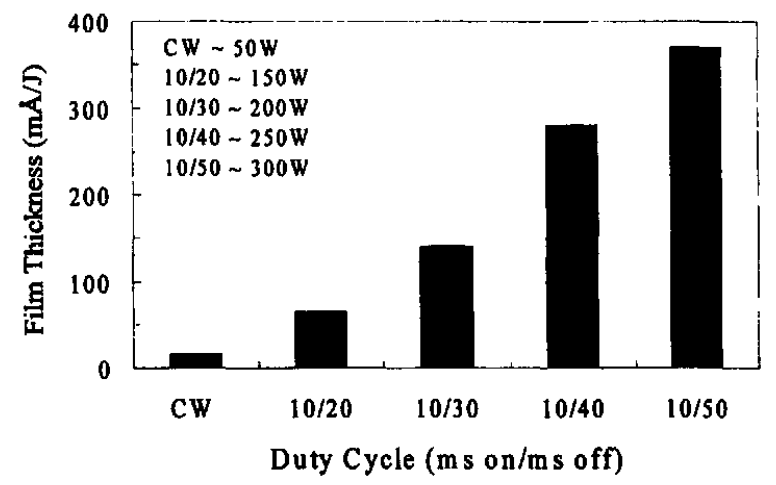

Figure 4. Film formation rates in $\mathrm{m} \AA / \mathrm{J}$ during $\mathrm{CW}$ and pulsed plasma polymerization of tetramethlysilane. All runs were carried out at an equivalent power input of $50 \mathrm{~W}$ 


\section{Discussion}

The results presented illustrate the high level of film chemistry controllability inherently available during the polymerization of monomers under pulsed plasma conditions. As shown for both perfluorohexane (Figure 1) and pyrrole (Figure 2), the film chemistry controllability available includes synthesis of relatively linear polymeric structures, as achieved under low duty cycle conditions. The dramatically increased percentage of $\mathrm{CF}_{2}$ groups in $\mathrm{C}_{6} \mathrm{~F}_{14}$ polymerization (Figure 1) and the sharply increased electrical conductivity of polypyrrole films obtained at the lower plasma duty cycles (Figure 3) are particularly dramatic examples of the highly organized, linear structures obtainable via this approach.

It is believed that there are a number of important changes in plasma dynamics under pulsed conditions which contribute to increased film chemistry controllability. As noted above, film formation rates per Joule of power input (Figure 4) provide evidence for polymer formation during plasma-off times. The formation of polymers during the plasma-off time occurs in the absence of substrate bias and vacuum UV photon fluxes. Both substrate bias and short wavelength photons have been shown to exert significant effects on film compositions under $\mathrm{CW}$ plasma conditions. In both cases, their influence is to randomize the film chemistry leading to enhanced cross-linking of the plasma polymers. Additionally, we have observed that substrate heating is generally far less pronounced during pulsed deposition than during $\mathrm{CW}$ polymerization, even when comparing runs of the same power inputs. The lower substrate temperatures maintained under pulsed conditions could promote more selective film formation chemistry, favoring processes having lower activation energies. All of these dynamic variables (i.e. substrate bias, photon flux, and substrate temperature) can contribute to formation of more highly linear polymeric structures under pulsed versus $\mathrm{CW}$ conditions. In particular, the ability to generate plasma polymers under exceptionally low power input conditions, as provided under pulsed condition, extends the synthesis of plasma polymers to highly ordered linear compositions which are simply not attainable under $\mathrm{CW}$ conditions.

\section{Conclusion}

The use of low duty cycle pulsed plasmas permits extension of synthesis of polymer films to lower W/FM values than available under $\mathrm{CW}$ conditions. This extension is of pivotal importance in synthesizing highly linear polymeric structures having specially desired properties, such as the high electrical conductivity shown in the present study.

\section{Acknowledgement}

We are pleased to acknowledge partial support of this research by the Texas Higher Education Coordinating Board, ATP Program, Grant \# 003656.

\section{References}

1. F. Denes, Trends Polym. Res., 5, 23 (1997)

2. H. Yasuda, Plasma Polymerization, Academic Press, Orlando, FL (1985)

3. C.R. Savage, R.B. Timmons, and J.W. Lin, Chem. Mater., 3, 575 (1991)

4. C.L. Rinsch, X. Chen, V. Panchalinam, R.C. Eberhart, J.W. Wang and R.B. Timmons, Langmuir, 12, 2995 (1996)

5. L.M. Han and R.B. Timmons, J. Poly. Sci,; Part A; Polym. Chem., 36, 3121 (1998)

6. M.E. Ryan, A.M. Hayes and J.P.S. Badyal, Chem. Mater., 8, 37 (1996)

7. J. G. Calderon and R. B. Timmons, Macromolecules, 31, 3216 (1998)

8. X. Chen, K. Rajeshwar, R.B. Timmons, J-J. Chen and O.M.R. Chyan, Chem. Mater., 8, 1067 (1996)

9. L.M. Han, K. Rajeshwar and R.B. Timmons, Langmuir, 13, 5941(1997)

10. N.M. Mackie, N.F. Dallesha, D.G. Castner and E.R. Fisher, Chem. Mater., 9, 349 (1997)

11. V. Panchalinam, X. Chen, C.R. Savage, R.B. Timmons and R.C. Eberhart, J. Appl. Polym. Sci., Appl. Polym. Symp., 54, 123 (1994)

12. J. Zhang, M. Wu, T. Pu, Z. Zhang, R. Jin, Z. Tong, D. Zhu, S. Cao, Thin Solid Films, 307, 14 (1997)

13. C.J. Cruz, J. Morales and R. Olayo, Thin Solid Films, 342, 119 (1999)

14. L.M. Han and R.B. Timmons, Chem. Mater. 10, 1422 (1998) 